\title{
Modulation of the inter-hemispheric asymmetry of motor-related brain activity using brain-computer interfaces
}

\author{
Michael Pereira ${ }^{1}$, Aleksander Sobolewski ${ }^{1}$ and José del R. Millán ${ }^{1}$
}

\begin{abstract}
Non-invasive brain stimulation has shown promising results in neurorehabilitation for motor-impaired stroke patients, by rebalancing the relative involvement of each hemisphere in movement generation. Similarly, brain-computer interfaces have been used to successfully facilitate movementrelated brain activity spared by the infarct. We propose to merge both approaches by using BCI to train stroke patients to rebalance their motor-related brain activity during motor tasks, through the use of online feedback. In this pilot study, we report results showing that some healthy subjects were able to learn to spontaneously up- and/or down-regulate their ipsilateral brain activity during a single session.
\end{abstract}

\section{INTRODUCTION}

An important area of research in neurorehabilitation deals with improving motor recovery after stroke by up- or downregulating the activity of one hemisphere through the use of non-invasive brain stimulation [1]-[3]. Two competing models have led to opposite recommendations, and the question whether excitability in the healthy hemisphere should be decreased to reduce its interhemispheric inhibition on the lesioned hemisphere [1], [4] or on the contrary, favoured to develop compensatory activity in the healthy hemisphere [5]. Recent models actually build on both previous rehabilitation schemes, tailoring the choice to individual patients, based on the amount of neural resources spared by the lesion [5]. The matter is further complicated by hemispheric specialisation: in healthy subjects, increased ipsilateral motor cortex involvement has been reported for precision-demanding motor tasks [6], [7] or finger tapping sequences of increased complexity [8], [9]. Such ipsilateral activity has been shown to exert a causal influence on task performance [10] and hemispheric asymmetries have been suggested to be related to augmented attention or executive control [11]. Taking these issues into account, an alternative, and arguably more adaptable and natural way for modulating interhemispheric rebalancing could be through the use of brain-computer interfaces (BCI) training.

BCI technology -allowing control and communication without the use of the peripheral nervous system has recently broadened its application areas of communication or motor substitution, to include motor recovery in the context of stroke rehabilitation [12], [13]. Indeed, when provided with real-time feedback of the BCI decoder, some users can learn to modulate their brainwaves to achieve better control, eventually inducing long term plastic changes in the central

\footnotetext{
${ }^{1}$ Michael Pereira, Aleksander Sobolewski and José; del R. Millán are with Chair in Non-invasive Brain-machine Interface, Ecole Polytechnique Fédérale de Lausanne, 1015 Lausanne, Switzerland michael.pereiradepfl.ch
}

nervous system (CNS) [14]. Recently, some research groups [15]-[17] have used BCI approaches to restore volitional motor control in hemiplegic patients by providing assistance (robotic arm/visual feedback) when movement intention is decoded, thus inducing activity-dependent CNS plasticity [14]. This approach promotes whatever salient brain signal features are present at the beginning of the therapy. We propose to consider BCI as a way to train brain signal features in a more controlled way, by rebalancing the relative involvement of each (hemi)motor cortex and on an individual basis.

(a) Representative trajectory

(b) Assistance
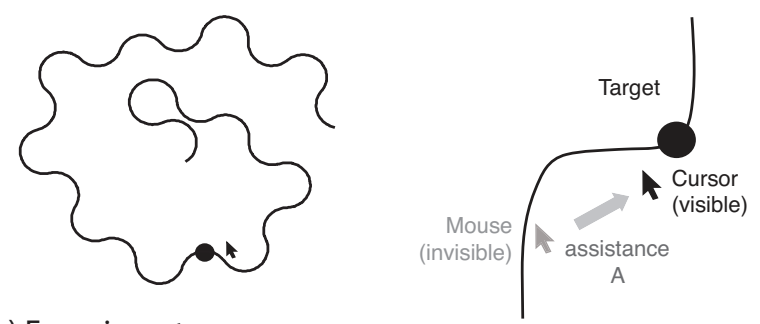

(c) Experiment

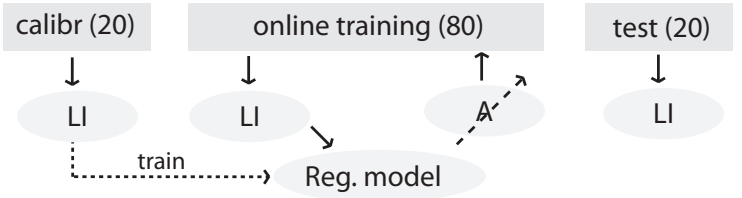

Fig. 1. a) An example trajectory composed of concatenated 50 pixels radius arcs of varied lengths. b) The implementation of the assistance: the cursor (visible) is displayed closer to the target centre, than would result purely from subject's movements with the mouse (invisible). c) The experiment is divided into three phases: lateralisation index (LI) values are recorded during the first 20-trial phase (calibr) and used to calibrate a regression model that is used during the 80-trial train phase (online), to convert LI instantaneous values into assistance " $A$ ", thus dynamically changing the difficulty of the task. Eventually, a 20-trial phase (test) was performed without assistance.

A first step towards this goal is to prove the feasibility for healthy subjects to wilfully modulate the lateralisation of their motor-related brain activity during purposeful movements, through the use of closed-loop BCI training. In this pilot study, we use a non-invasive BCI paradigm based on an electrophysiological marker of the asymmetry of neural activity from motor cortices -indexed by sensorimotor rhythms (SMR) [18]. This so-called lateralisation index (LI) ${ }^{1}$ is used to provide online feedback while performing a

\footnotetext{
${ }^{1}$ Such indices of asymmetry have already been suggested for prognostics of stroke outcome [19].
} 
visuomotor tracking task, by dynamically adapting the task difficulty; promoting ipsilateral lateralisation by rewarding ipsilateral LI by lower task difficulty (further referred to as the "ipsi scenario") or promoting contralateral lateralisation by rewarding a shift of the LI towards the contralateral hemisphere by lower task difficulty (the "contra scenario"). Preliminary results show that during a single short training session (less than one hour), one out of four subject was able to lateralise his brain activity both towards the contralateral (in the contra scenario) and ipsilateral (in the ipsi scenario) hemisphere and two subjects were only able to lateralise their brain activity towards the ipsilateral hemisphere.

\section{METHODS}

\section{A. Experimental protocol}

Four healthy right-handed subjects participated in two visuomotor tracking experiments. Subjects provided written informed consent beforehand and the experiments were part of a study approved by the local ethics committee. One experiment was meant to promote ipsilateral shift (the ipsi scenario) and another to promote contralateral shift of activity (the contra scenario). Subjects 1 and 2 started with the ipsi experiment, then performed the contra experiment after an interval of minimum one week, while subjects 3 and 4 did the experiments in a reversed order. The task consisted in using the computer mouse to follow a target (a red circle with 10 pixels radius), moving at constant speed along a complex, twisting trajectory for 20 seconds (Fig. 1a). The entire trajectory was visible at all times during the 20 $\mathrm{s}$ trial. For every trial, the trajectory was precomputed by concatenating arcs of 50 pixels radius and varied lengths, with the whole trajectory subtending about $10^{\circ}$ of visual angle. A feedback score based on average performance was shown at the end of each trial. Each experiment consisted of three phases (Fig. 1c). In the first "calibration phase", subjects performed twenty trials; EEG data from this phase was used to calculate their spontaneous lateralisation index (LI; see below). In the subsequent "online training phase", the subjects performed the visuomotor tracking again, but at the same time were required (and incentivised) to modulate their LI. The LI values were being fed back to them in real-time through instantaneous task difficulty, which was manipulated by providing assistance "A". The assistance caused the cursor to be displayed closer to the target's centre than would result purely from subject's movements with the mouse (Fig. 1b), reducing the effect of subjects' errors. At $A=0 \%$ assistance, the computer mouse fully controlled the cursor while at $A=100 \%$ assistance, the cursor would automatically follow the target, independently from the movement of the computer mouse. During the online phase, $A$ was kept in the [0\%, $80 \%]$ interval. Depending on $A$, the colour of the target would vary from red $(A=0 \%)$ to green $(A=80 \%)$.

The assistance was dynamically adjusted based on the instantaneous value of the LI: $A=\alpha_{1} * L I+\alpha_{0}$, where $\alpha_{1}$ and $\alpha_{0}$ are scalars computed based on the distribution of the LI during the calibration phase (Fig. 2): when promoting ipsilateral shift of motor activity, $\alpha_{1}$ and $\alpha_{0}$ were set so that the $10 \%$ most ipsilateral part of the LI distribution $\left(P_{90}: 90 t h\right.$ percentile) would provide maximum assistance $(A=80 \%)$ while the $10 \%$ most contralateral part of the LI distribution ( $P_{10}: 10 t h$ percentile) would provide minimum assistance ( $A=0 \%$ ). Conversely, when promoting a contralateral shift of activity, $\alpha_{1}$ and $\alpha_{0}$ were set so that the $10 \%$ most contralateral part of the LI distribution $\left(P_{10}: 10 \mathrm{th}\right.$ percentile) would provide maximum assistance $(A=80 \%)$ and the $10 \%$ most ipsilateral part of the LI distribution $\left(P_{90}: 90 t h\right.$ percentile) would provide minimum assistance $(A=0 \%)$. In between, $A$ would increase/decrease linearly. The online training phase consisted of 80 trajectories (trials) and was followed by a last "test phase" of 20 trials without any assistance.
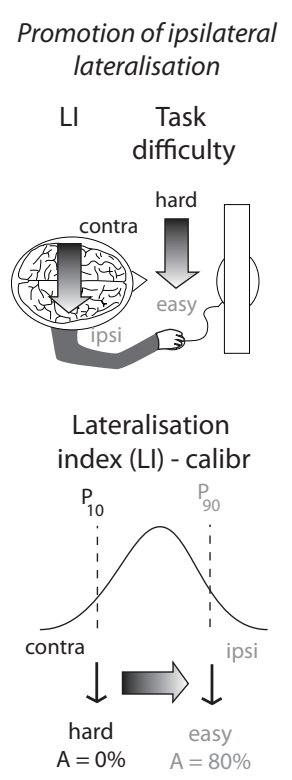

\section{Promotion of contralateral lateralisation

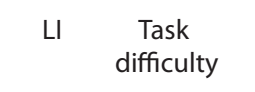

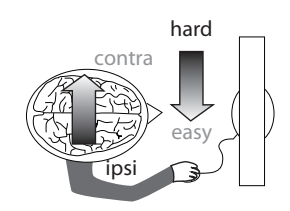

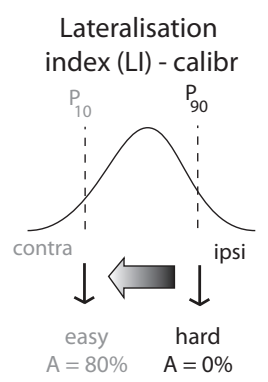

Fig. 2. Two different online training scenarios. Left: when promoting ipsilateral shift in motor-related brain activity (ipsi scenario), the more ipsilateral activity compared to contralateral activity, the more assistance was given. The regression model was calibrated such that the $10 \%$ most contralateral part ( $P_{10}: 10 t h$ percentile) of the LI distribution would correspond to no assistance $(\mathrm{A}=0 \%$, difficulty: hard) while the $10 \%$ most ipsilateral part ( $P_{90}: 90 t h$ percentile) of the LI distribution would correspond to maximum assistance ( $\mathrm{A}=80 \%$, difficulty: easy). Right: when promoting contralateral shift in motor-related brain activity (contra scenario), the more ipsilateral activity compared to contralateral activity, the less assistance was given. The regression model was calibrated such that the $10 \%$ most contralateral part ( $P_{10}: 10 t h$ percentile) of the LI distribution would correspond to maximum assistance (A=80\%, difficulty: easy) while the $10 \%$ most ipsilateral part (P90: 90th percentile) of the LI distribution would correspond to no assistance ( $\mathrm{A}=0 \%$, difficulty: hard) .

\section{B. Data acquisition and processing}

EEG activity was registered using a 64 electrode Biosemi ActiveTwo system (BioSemi B.V., the Netherlands) in an extended 10-20 montage at $2048 \mathrm{~Hz}$ sampling rate, downsampled to $256 \mathrm{~Hz}$ and re-referenced to a common average reference. Consecutive one second long windows were extracted from the $\mathrm{C} 3$ and $\mathrm{C} 4$ sensors (overlying the hand representations of the primary motor cortex for each hemisphere [20]), the DC component was removed (by subtraction of the mean EEG over the window) and power spectrum 
was computed using the Welch method (with five $500 \mathrm{~ms}$ Hamming windows of $75 \%$ overlap).

Lateralisation indices were computed for $f=\mu$ (10-12 $\mathrm{Hz})$ and $f=\beta(18-22 \mathrm{~Hz})$ frequency bands as follows:

$$
L I^{f}=\left(P_{C 3}^{f}-P_{C 4}^{f}\right) /\left(P_{C 3}^{f}+P_{C 4}^{f}\right)
$$

with $P_{e}^{f}$ being the average power for electrode $e$ in frequency band $f$ ). The final LI was the mean of $L I^{\mu}$ and $L I^{\beta}$ and had a theoretical range between -1 for maximal contralateral desynchronisation and 1 for maximal ipsilateral desynchronisation (note that higher brain activation corresponds to lower SMR power and vice versa). Electrooculographic (EOG) signals were registered with three extra electrodes placed above the nasion and below the outer canthi of the eyes. Vertical EOG activity was calculated as the difference between the signal from the central EOG electrode and the mean of the signals from the lateral EOG electrodes; horizontal EOG activity was calculated as the difference between the signals from the lateral EOG electrodes. Automated detection of vertical or horizontal EOG amplitude exceeding $70 \mu \mathrm{V}$ was used to reject all time windows contaminated with eye movement artifacts. When a window was rejected, its value was simply substituted by the previous LI value. For online processing, the LI was smoothed with a first order lowpass IIR filter (with a coefficient of 0.8), then transformed to assistance coefficients $A$ using the pre-computed $\alpha_{1}$ and $\alpha_{0}$.

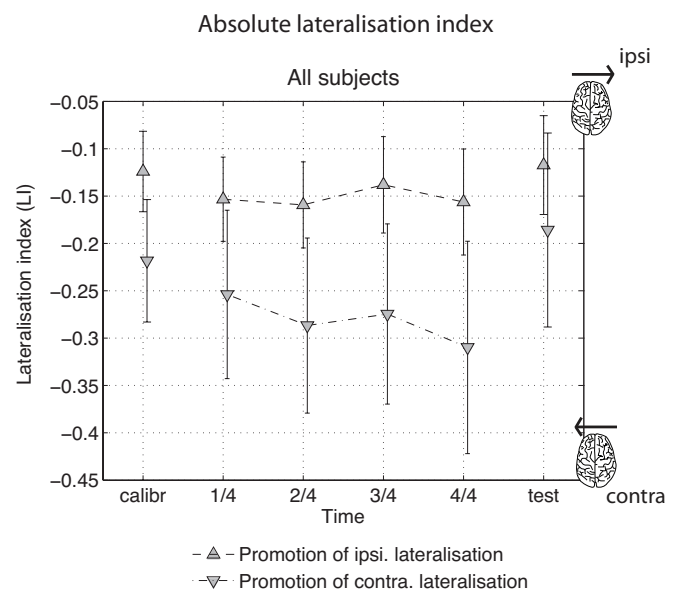

Fig. 3. Lateralisation index (LI) when promoting ipsilateral lateralisation (upward triangle) or contralateral lateralisation (downward triangle) for the whole experiment: calibration phase (calibr), online training phase $(1 / 4,2 / 4$, $3 / 4,4 / 4)$ and test phase (test). Whiskers indicate standard error of the mean.

\section{RESULTS}

In the following analysis, the LI were recomputed post-hoc and averaged per trial, then averaged per block of 20 trials. This led to one 20-trial block for the calibration phase, four 20-trial blocks for the online training phase and one 20-trial block for the test phase. Standard errors of the mean were computed across the blocks.

First of all, some (non-significant) tendency towards a contralateral lateralisation during the online training phase, compared to the calibration and test phases can be seen, regardless of the scenario (ipso or contra) given to the subject (Fig. 3). That is due to the fact that assistance was provided (dynamically) only during the online training phase, rendering the task easier during the time intervals when assistance was high, which can itself contribute to a reduction of the ipsilateral activation [6], [7] and thus a contralateral lateralisation.

However, to actually analyse the modulation of the lateralisation index during the online training phase itself, we compared trial-averaged LI from the first block to those from the subsequent three blocks. For this purpose, a relative LI was computed by subtracting the mean LI in the first block to the mean LI of the subsequent three blocks (Fig. 4). We considered a subject to have managed to lateralise his motor-related brain activity when trial-averaged LI in at least one out of the three last blocks was significantly (unpaired T-test, $p<0.05$ ) shifted towards the correct hemisphere. Subject 1 was able to lateralise his brain activity towards both ipsilateral and contralateral hemispheres. Subject 3 and 4 were only able to lateralise their brain activity towards the ipsilateral hemisphere while subject 2 was not capable to lateralise his brain activity and even had contralateral lateralisation when ipsilateral lateralisation was promoted.

\section{Lateralisation index relative to the first training block}
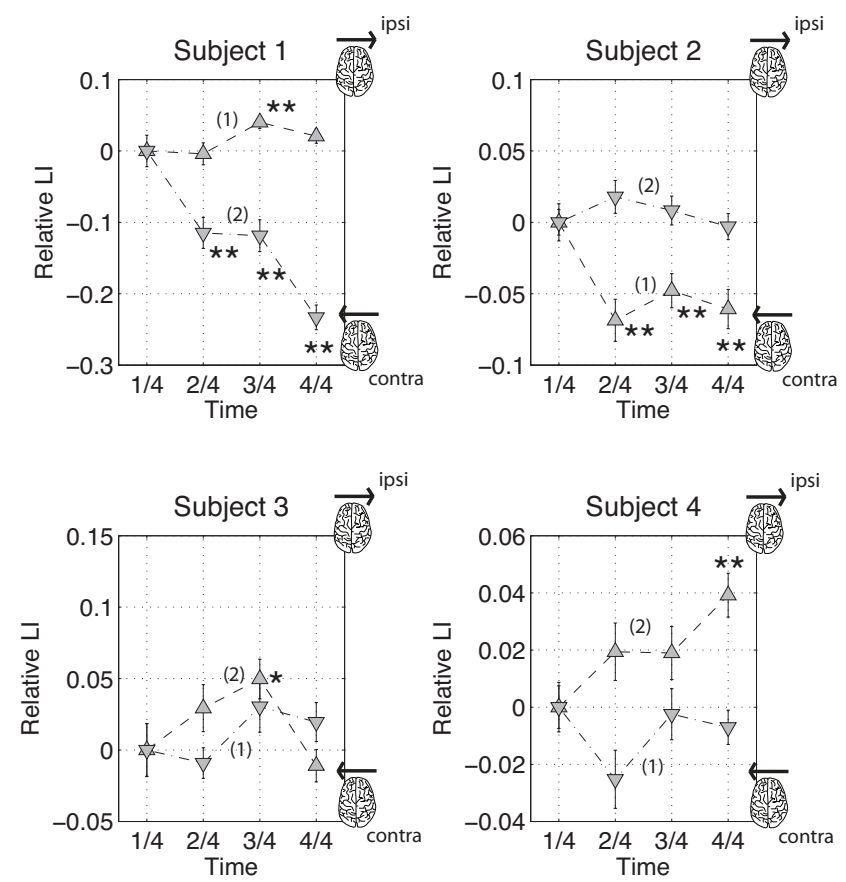

$-\otimes-$ Promotion of ipsi. lateralisation
$-\nabla-$ Promotion of contra. lateralisation

$$
\begin{array}{cc}
* & p<0.05 \\
\text { * } & p<0.01
\end{array}
$$

Fig. 4. Lateralisation index (LI), relative to the first 20 trials of the online training phase, when promoting ipsilateral lateralisation (upward triangle) or contralateral lateralisation (downward triangle). Whiskers indicate standard error of the mean. Single stars appear next to 20-trial blocks that were significantly (unpaired T-test, $p<0.05$ ) different from the first block. Double stars represent a $p<0.01$ significance. (1) represents the first experiment (in time) while (2) represent the second experiment. 


\section{DISCUSSION}

In this pilot study, we found that at least some subjects could successfully lateralise their motor-related brain activity (indexed by SMR desynchronisation) towards ipsilateral or contralateral motor areas using a BCI training paradigm. The study has a number of limitations: as yet, a small number of subjects with considerable inter-subject differences, absence of brain lesions (healthy subjects) and the probable influence of other factors like the level of performance and/or the effects of learning. However, we argue that BCI, after further thorough validation, could be used as a more natural alternative to electric or magnetic brain stimulation for interhemispheric rebalancing during stroke rehabilitation of motor function, with the advantage of taking place during rehabilitation exercises. The low amount of online training (single session, less than an hour) could be the reason for the absence of lateralisation of some subjects and it might be that a "healthy", i.e. functional hemispheric balance of motor activity could be more difficult to modulate than for abnormal hemispheric asymmetry, as can be seen in stroke patients. Additional lesser advantages include a short calibration phase, needing less than 7 minutes of EEG recordings and the limited number of electrodes involved in the computation of the LI. Finally, such BCI paradigms could be used to glean interesting neuroscientific knowledge about the nature of brain oscillations (SMR) in producing functional movements, and their possible modulation by topdown factors.

\section{REFERENCES}

[1] U. Ziemann, "Improving disability in stroke with RTMS.," Lancet Neurology, vol. 4, no. August, pp. 454-455, 2005.

[2] F. C. Hummel and L. G. Cohen, "Non-invasive brain stimulation: a new strategy to improve neurorehabilitation after stroke?," Lancet Neurology, vol. 5, no. August, pp. 708-712, 2006.

[3] F. Fregni and A. Pascual-Leone, "Technology insight: noninvasive brain stimulation in neurology-perspectives on the therapeutic potential of rTMS and tDCS.," Nature clinical practice. Neurology, vol. 3, pp. 383-393, 2007.

[4] N. Murase, J. Duque, R. Mazzocchio, and L. G. Cohen, "Influence of Interhemispheric Interactions on Motor Function in Chronic Stroke.," Annals of Neurology, vol. 55, pp. 400-409, 2004.

[5] G. Di Pino, G. Pellegrino, G. Assenza, F. Capone, F. Ferreri, D. Formica, F. Ranieri, M. Tombini, U. Ziemann, J. C. Rothwell, and V. Di Lazzaro, "Modulation of brain plasticity in stroke: a novel model for neurorehabilitation," Nature Reviews Neurology, vol. 10, no. 10, pp. 597-608, 2014.
[6] R. D. Seidler, D. C. Noll, and G. Thiers, "Feedforward and feedback processes in motor control.," NeuroImage, vol. 22, pp. 1775-83, Aug. 2004.

[7] C. M. Buetefisch, K. P. Revill, L. Shuster, B. Hines, and M. Parsons, "Motor demand-dependent activation of ipsilateral motor cortex.," Journal of neurophysiology, vol. 112, pp. 999-1009, Aug. 2014.

[8] F. Hummel, R. Kirsammer, and C. Gerloff, "Ipsilateral cortical activation during finger sequences of increasing complexity: representation of movement difficulty or memory load?," Clinical Neurophysiology, vol. 114, pp. 605-613, Apr. 2003.

[9] T. Verstynen, J. Diedrichsen, N. Albert, P. Aparicio, and R. B. Ivry, "Ipsilateral motor cortex activity during unimanual hand movements relates to task complexity.," Journal of neurophysiology, vol. 93, pp. 1209-22, Mar. 2005.

[10] S. Y. Schaefer, K. Y. Haaland, and R. L. Sainburg, "Dissociation of initial trajectory and final position errors during visuomotor adaptation following unilateral stroke.," Brain research, vol. 1298, pp. 78-91, Nov. 2009.

[11] D. J. Serrien, R. B. Ivry, and S. P. Swinnen, "Dynamics of hemispheric specialization and integration in the context of motor control.," Nature reviews. Neuroscience, vol. 7, pp. 160-6, Feb. 2006.

[12] K. Ang and C. Guan, "Brain-Computer Interface in Stroke Rehabilitation," Journal of Computing Science and Engineering, vol. 7, no. 2, pp. 139-146, 2013.

[13] J. d. R. Millán, R. Rupp, G. R. Müller-Putz, R. Murray-Smith, C. Giugliemma, M. Tangermann, C. Vidaurre, F. Cincotti, A. Kübler, R. Leeb, C. Neuper, K.-R. Müller, and D. Mattia, "Combining BrainComputer Interfaces and Assistive Technologies: State-of-the-Art and Challenges.," Frontiers in Neuroscience, vol. 4, Jan. 2010.

[14] J. J. Daly and J. R. Wolpaw, "Brain-computer interfaces in neurological rehabilitation.," Lancet Neurology, vol. 7, pp. 1032-43, Nov. 2008.

[15] A. Ramos-Murguialday, D. Broetz, M. Rea, L. Läer, O. Yilmaz, F. L. Brasil, G. Liberati, M. R. Curado, E. Garcia-Cossio, A. Vyziotis, W. Cho, M. Agostini, E. Soares, S. Soekadar, A. Caria, L. G. Cohen, and N. Birbaumer, "Brain-machine interface in chronic stroke rehabilitation: A controlled study.," Annals of Neurology, vol. 74, pp. 100-8, July 2013.

[16] F. Pichiorri, G. Morone, M. Petti, J. Toppi, I. Pisotta, M. Molinari, S. Paolucci, M. Inghilleri, L. Astolfi, F. Cincotti, and D. Mattia, "Brain-computer interface boosts motor imagery practice during stroke recovery.," Annals of Neurology, 2015.

[17] A. Biasiucci, R. Leeb, A. Al-Khodairy, V. Buhlmann, and J. d. R. Millán, "Motor recovery after stroke by means of BCI-guided Functional Electrical Stimulation.," in 5th International BCI Meeting, p. 49, 2013.

[18] G. Pfurtscheller, A. Stancák, and C. Neuper, "Event-related synchronization (ERS) in the alpha band - An electrophysiological correlate of cortical idling: A review.", International Journal of Psychophysiology, vol. 24 , pp. 39-46, 1996.

[19] R. V. a. Sheorajpanday, G. Nagels, A. J. T. M. Weeren, M. J. a. M. van Putten, and P. P. De Deyn, "Quantitative EEG in ischemic stroke: Correlation with functional status after 6 months.," Clinical neurophysiology, vol. 122, pp. 874-83, May 2011.

[20] G. Pfurtscheller and a. Berghold, "Patterns of cortical activation during planning of voluntary movement.," Electroencephalography and clinical neurophysiology, vol. 72, pp. 250-8, Mar. 1989. 